\title{
Lightness models, gradient illusions, and curl
}

\author{
LAWRENCE E. AREND and ROBERT GOLDSTEIN \\ Eye Research Institute of Retina Foundation, Boston, Massachusetts, \\ and Harvard Medical School, Boston, Massachusetts
}

\begin{abstract}
Gradient illusions require that models of suprathreshold appearance include a spatial integration that fills areas between edges. We describe a structural problem inherent in such models; for many scenes there are inconsistencies (nonzero curl) in thresholded derivatives that prevent simple spatial integration. Our experiments show that the human visual system does encounter curl problems and that it uses two different types of perceptual solution: field segmentation and lightness-gradient manipulation. The latter occurs under conditions where field segmentation is impossible. At least two such conditions can occur: failure to form a segmenting contour and topological problems in potential segmenting contours.
\end{abstract}

\section{THEORY}

\section{Gradient Illusions and Models Explaining Them Gradient Illusions}

An adequate model of lightness ${ }^{1}$ and color appearance in general suprathreshold scenes must be able to explain a class of illusions that we will refer to as gradient illusions. The most familiar of these illusions is known as the Cornsweet illusion (Cornsweet, 1970). In this paper we describe models that include features required to explain gradient illusions. We then analyze mathematical problems those features produce in the models. The difficulties are not restricted to predictions for obscure illusory patterns; they occur in many natural scenes. Finally, we describe experiments with new gradient illusions that make even greater demands of candidate models.

At high contrast, a radial sawtooth luminance pattern has a spatial lightness distribution roughly isomorphic with its luminance distribution: Steep and shallow luminance gradients are seen as steep and shallow lightness gradients. At lower contrasts, however, the shallow gradient produces little (if any) lightness gradient, leaving an apparent step distribution of lightness (Figure 1). The luminances at corresponding locations within the three cycles (e.g., the centers) are equal, but the lightnesses are not.

A number of related illusions can be constructed from various combinations of shallow and steep luminance gradients (Arend, Buehler, \& Lockhead, 1971; Cornsweet, 1970; Craik, 1966; O'Brien, 1958).

\section{Spatial Differentiation}

It is widely believed (e.g., Campbell, Howell, \& Robson, 1971; Cornsweet, 1970; Ratliff \& Sirovich, 1978)

This work was supported by Contract F49620-83-C-0052 from the Air Force Office of Scientific Research to the first author. Some of this work was reported at the Spring 1985 meeting of the Association for Research in Vision and Ophthalmology. Address correspondence to Lawrence E. Arend, Jr., Eye Research Institute of Retina Foundation, 20 Staniford Street, Boston, MA 02114. that these illusions are fully explained by the visual system's insensitivity to shallow gradients, the same insensitivity that produces the low-frequency decline of the spatial contrast sensitivity function (CSF). The argument is sketched in Figure 2.

A step distribution of luminance and a sawtooth luminance distribution, for example, differ primarily in low spatial frequencies-the step can be analyzed into the sum of a sawtooth and a shallow gradient. After low-frequency attenuation by the visual system, responses to the two patterns are roughly equivalent. ${ }^{2}$

This, however, is only half an explanation. According to the argument, the three regions separated by the two sharp luminance steps should be identical in appearance. In practice, both casual observation and laboratory measurements (reported below) show that the centers of the three cycles differ significantly in lightness. Under some conditions a matching field must be twice as luminous to match one cycle of sawtooth as to match another (Arend, 1987). Similar data have been reported for the Cornsweet illusion (Dooley \& Greenfield, 1977). Some spatial integration process is required to fill in between edge transients.

If the low-spatial-frequency information were merely attenuated in thresholding, rather than lost, then the integration would restore the different appearances of the sawtooth and step. The nearly identical appearances of the patterns requires true thresholding.

\section{Integration Models}

There are a number of visual phenomena other than gradient illusions that require some kind of filling-in between edges (see Arend, 1973, for a critical review). ${ }^{3}$ Stabilized retinal image experiments (Arend, 1973; Gerrits, de Haan, \& Vendrik, 1966; Krauskopf, 1963) show that filling-in between edges is a large effect, playing a large role in normal perception, where temporal changes of retinal illuminance and chrominance are often confined to the immediate vicinity of edges (Walls, 1954). 


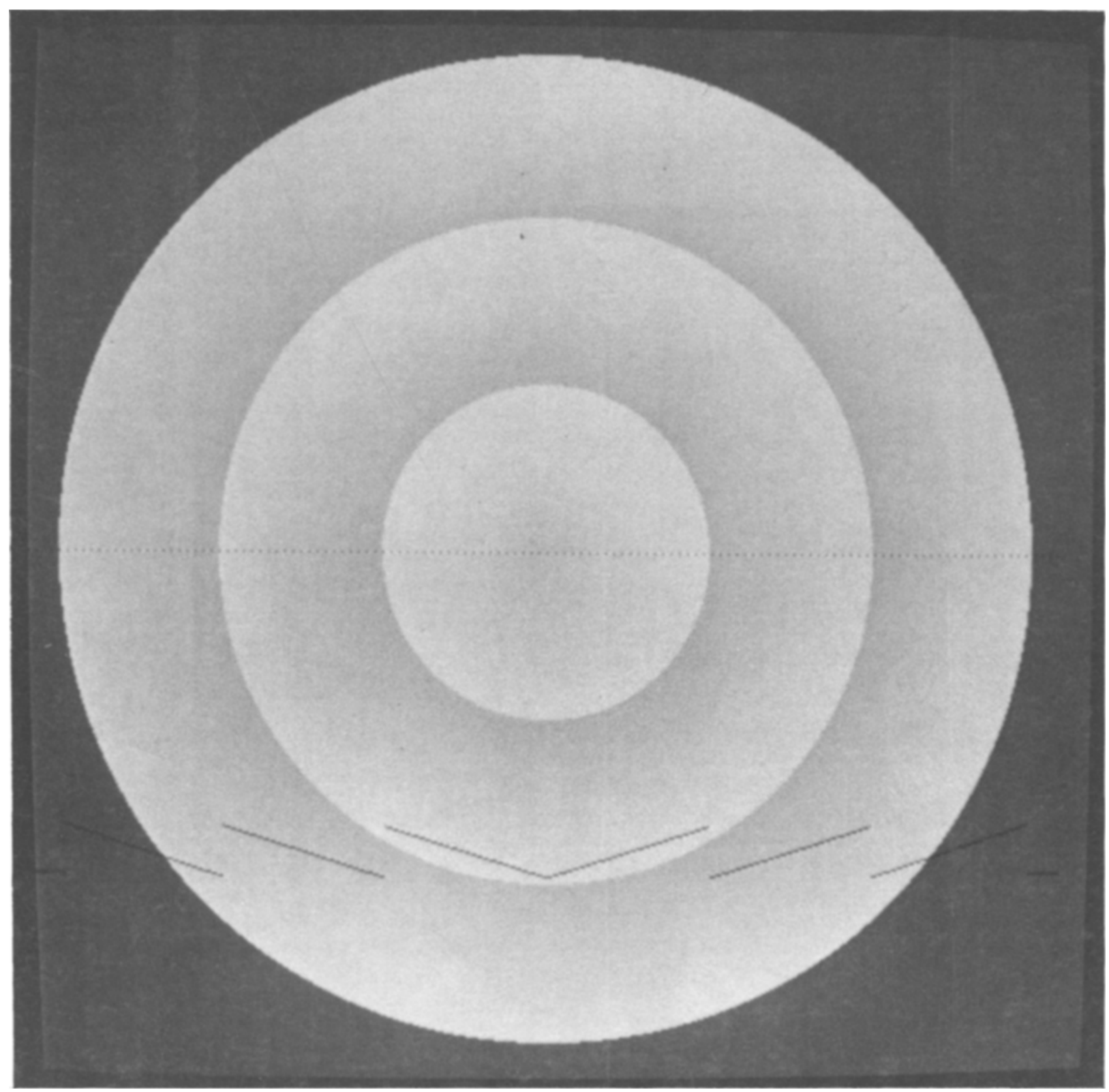

Figure 1. Radial sawtooth luminance pattern. Luminance distribution is three identical cycles of sawtooth, increasing outward along a radius. Luminances at corresponding points in the cycles are equal. The pattern should be viewed from as close as possible.

\section{Local Versus Global Integration}

Several authors have proposed local filling-in within closed contours (Cohen \& Grossberg, 1984; Davidson \& Whiteside, 1971; Fry, 1948; Gerrits et al., 1966). Models using local (contour-bounded) filling-in do not easily explain phenomena in which information from one contour extends beyond the next, for example, multiple-cycle gradient illusions (like that shown in Figure 1) and multipleedge stabilized patterns (Yarbus, 1967). ${ }^{4}$

Although a number of authors have recognized a need for global integration of edge information, only four models have been described in enough detail to permit analysis of their mathematical properties. Of these, two use 1-D algorithms (Arend, 1973; Land \& McCann, 1971) and two use 2-D algorithms (Blake, 1985; Horn, 1974). We will hereafter refer to these four models as integration lightness models.

\section{One-Dimensional Models}

The details of the two 1-D models differ, but the basic algorithm for encoding spatial luminance change in temporally constant displays is the same. Figure 3 gives an outline of the calculation of the relative lightnesses of any two points $A$ and $B$. Throughout this paper we assume that optical (and possibly neural) blur produces a retinal illuminance distribution with no discontinuities. The first 


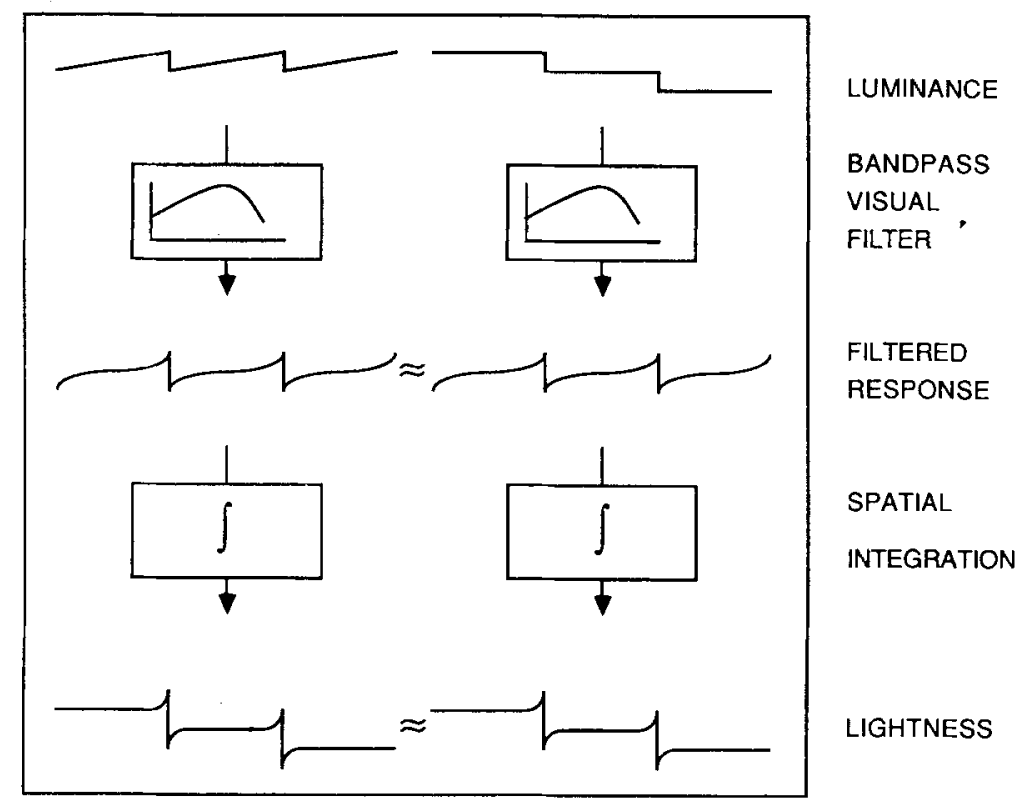

Figure 2. Sketch of low-frequency-attenuation argument. Attenuation alone predicts similar appearance, but the wrong appearance. Adding an integration stage produces the observed appearance.

step takes the logarithm of local retinal illuminance. Next the derivative of log illuminance along a line between the two points (i.e., the component of the gradient in the direction of the line) is computed. The derivative is then thresholded by weighting it with a function giving zero weight to small derivatives and unit weight to large derivatives. The integral of these thresholded derivatives along the line gives the lightness of point B relative to point A.

For our present purposes the details of the process taking the spatial derivative are irrelevant; any oddsymmetric linear mechanism with frequency sensitivity such as that shown in Figure 2 (i.e., unit slope over low spatial frequencies) will do. Examples are Land and McCann's (1971) spatial difference, Arend's (1973) temporal-change mechanism, or the summed output of a set of odd-symmetric, medium-bandwidth spatialfrequency filters with a sensitivity envelope as shown in Figure 2. The temporal-change mechanism has been offered as an explanation of the relative sensitivities of medium-bandwidth spatial channels (Arend, 1976a, 1976b).

All of these arguments inappropriately apply a 1-D analysis to one dimension of a 2-D pattern. The argument outlined in Figure 2 and the Arend (1973) model ignore 2D problems altogether by considering only patterns with luminance change along a single dimension. Land and his coworkers addressed the problem of 2-D patterns by computing lightness as a nonlinear average of results over multiple paths. The paths chosen for the computation are a subset of the infinitude of possible paths between each pair of points sampled from the infinitude of possible pairs of endpoints (Land, 1986).

\section{Two-Dimensional Models}

Second derivatives versus first derivatives. Horn's (1974) 2-D model was based on a second-derivative operator rather than the gradient. His operator has the computational advantage of being isotropic, yielding a nondirectional, scalar descriptor of local luminance change (more precisely, of spatial luminance acceleration). This isotropy is, however, a liability with respect to modeling human spatial vision: There is a great deal of psychophysical evidence of orientation tuning in human pattern vision, and the process of acquiring edge information through eye movements is inherently directional.

Perhaps even more important, true second-derivative operators are by definition insensitive to linear gradients. Horn's (1974) model avoids losing first-order pattern information only by requiring that the viewed scene be completely surrounded by a uniform field. This "special viewpoint" requirement severely limits the algorithm's utility for modeling the human visual system. We therefore need a first-derivative model.

A simple two-dimensional model. In the 2-D retinal illuminance distribution, spatial illuminance change is a vector field, the gradient of illuminance, possessing a direction as well as a magnitude. An appropriate algorithm should therefore deal directly in vector quantities.

Arend (1985) and Blake (1985) proposed a simple 2-D model, which is outlined in Figure 4. The model is described here in informal terms (for a more concise and rigorous mathematical presentation, see Blake, 1985). The gradient of $\log$ retinal illuminance is thresholded in the same manner as the threshholding of derivatives in the 1-D model, that is, by a weighting function that goes from 

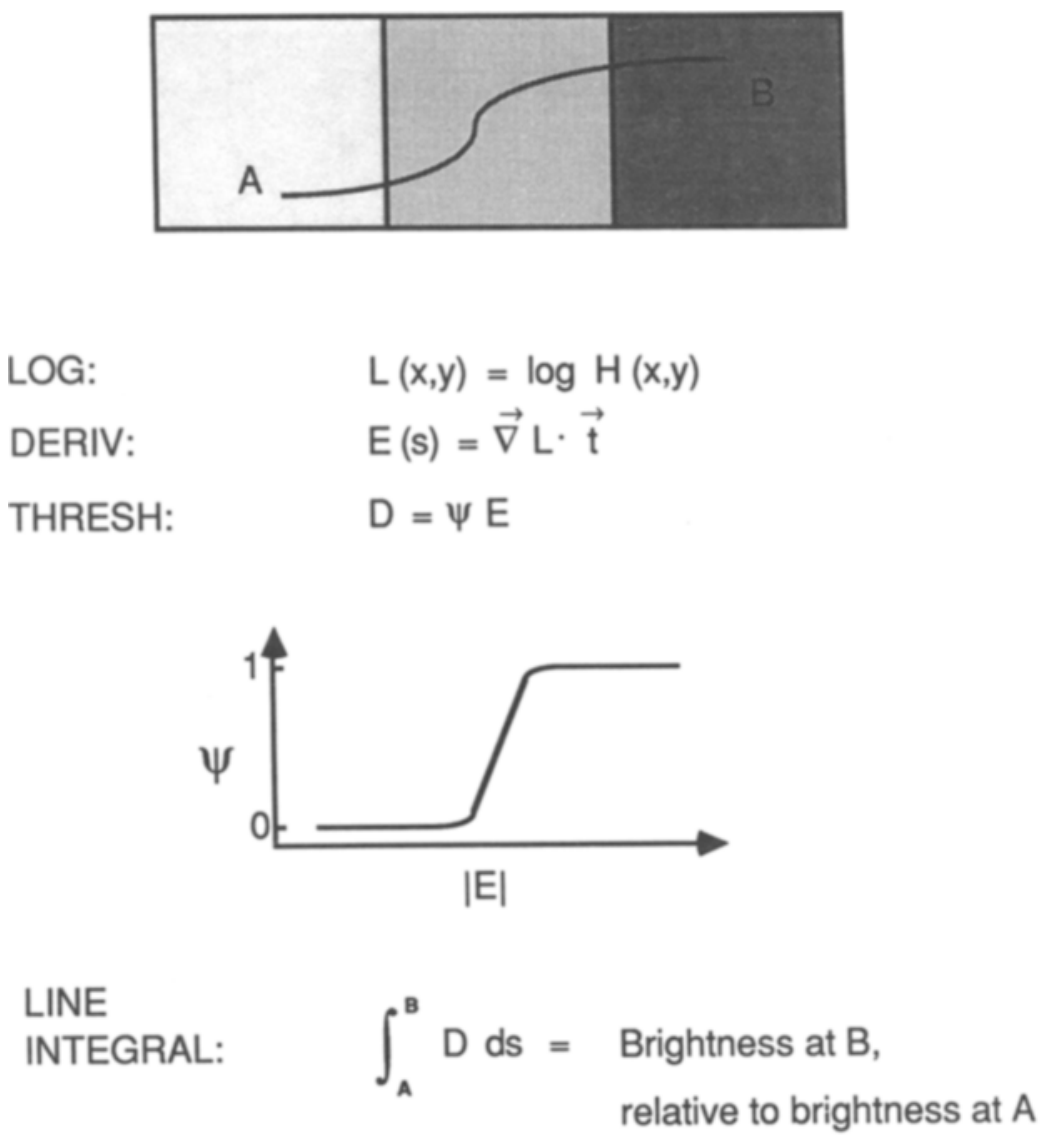

\section{Problem: Value of integral is generally path-dependent due to threshold.}

Figure 3. Sketch of algorithms of 1-D models. Models take the first spatial derivative of $\log$ retinal illuminance along a line. The result is thresholded by multiplication with a scalar function of the derivative magnitude, and the result is integrated along the line to obtain relative lightnesses of the endpoints. The threshold nonlinearity makes the value of the integral depend on the path chosen from $A$ to $B$.

zero to one as a function of the amplitude of the local gradient. This thresholded gradient is then treated as the gradient of the desired response, the lightness field. The principal advantage of the 2-D model over the 1-D models is that it represents 2-D spatial changes directly, rather than deriving them from multiple 1-D changes. The awkward line-integral analysis of the 1-D models is avoided completely.

Our computational model in its current form is a direct implementation of this model (see Appendix for details). The local compression, gradient, and threshold processes are straightforward algorithms manipulating local data and local differences.

For some patterns, integration over the thresholded gradient is also straightforward. For other patterns, integra- tion is impossible without special procedures, which will be discussed at length later in this paper. In the simple case, the integration algorithm simply constructs the output pattern pixel by pixel by summing the $x$ and $y$ components of the thresholded gradient. Figure 5 shows a sample calculated response for this simple case, the response for the radial sawtooth distribution of Figure 1.

\section{Inconsistent Integrals}

\section{Integration Problems}

Interposition of the threshold nonlinearity between the differentiation and integration produces severe problems for the integration process. The problem is perhaps more easily illustrated with the 1-D algorithm of Figure 3 than with the 2-D model, which requires concepts new to vi- 
LOG:

GRAD:

THRESH:

$$
L=\log H(x, y)
$$$$
\vec{E}=\vec{\nabla} L
$$

$$
\vec{D}=\psi \vec{E}
$$

$$
\psi=f(|\vec{E}|)
$$

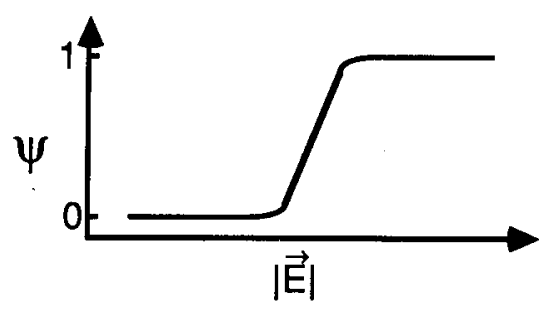

INTEGRAL: $\quad$ Given $\quad \vec{\nabla} R=\vec{D} \quad$ find $R$

\author{
Problem: Due to nonlinearity of threshold $\vec{D}$ not \\ necessarily gradient of anything, i.e., the \\ integral need not exist.
}

Figure 4. Sketch of algorithms of 2-D models. Models take the gradient of log retinal illuminance. The result is thresholded by multiplication with a scalar function of the gradient magnitude, and the result is integrated to obtain a field of predicted lightnesses. The threshold nonlinearity requires a complicated integration algorithm since the thresholded gradient field is not, in general, a gradient.

sion research. Keep in mind, however, that our current model is the 2-D one and that it integrates over areas rather than lines.

Expressed in terms of the 1-D model, the problem is that integration of thresholded derivatives from a point, $A$, to another point, $B$, along different paths will, in general, give conflicting answers for the relative lightnesses of A and B. In Figure 6a, on the left, is a diagram of a simple pattern with inconsistent line integrals, three cycles of a shallow luminance sawtooth on a dark surround. $A$ and $B$ are at corresponding points in their cycles and therefore have the same luminance. The line integral along path 1 is shown schematically on the right. The shallow gradient of the sawtooth is below threshold, so only the two abrupt increases contribute to the integral. The integral indicates that $B$ is lighter than $A$. The lower curve shows the line integral along path 2 . The sawtooth is uniform vertically, so the first suprathreshold luminance change is at the upward step onto the background. Con- tinuing around the path, the background is uniform, there is a downward step onto the sawtooth equal to the upward step, and there is no further change to $B$. Therefore, the integral along path 2 says that $A$ and $B$ have the same lightness, contradicting path 1 . Stated differently, the circulation integral along one path from $A$ to $B$ and then back to $A$ on the other path is not zero. This is inherently inconsistent, indicating that the lightness at $A$ is not equal to the lightness at $A$.

The inconsistencies cannot readily be handled by averaging line integrals from multiple paths. In a recent version of Land's (1986) model, the response at a particular point, $i$, in the image is the average of many line integrals starting at various points and proceeding along various paths to $i$. For patterns with path-dependent line integrals, this algorithm's predicted lightness distribution depends critically on sampling of start points and paths. To date little analysis of path selection in the Land model has been published. 


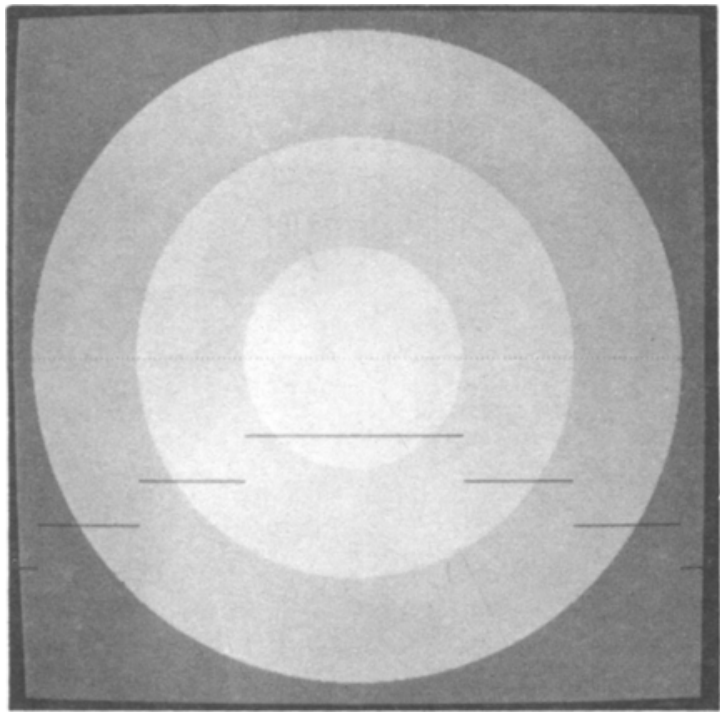

Figure 5. Predicted lightness field in a simple case (radial sawtooth luminance distribution, Figure 1). The shallow luminance gradient of the sawtooth is given a weight of zero in the psi-function stage.

\section{Curl}

Substituting the 2-D gradient model for the 1-D model does not avoid the problem introduced by the threshold nonlinearity. For patterns giving inconsistent line integrals, the thresholded gradient field is not a gradient at all. The structure of the vector field formed by thresholding the gradient is inconsistent with its being the gradient of any scalar field (in particular, any log retinal image).

The 2-D expression of the inconsistency is a vector quantity, curl, defined below. For a field to be a gradient (and have an integral), the curl of the field must be zero at all points (Blake's [1985] Equation 5). The close relationship between inconsistent line integrals (nonzerocirculation integrals) and curl can be seen from the differential definition of curl: The magnitude of the curl of a 2-D field at a point $(x, y)$ is just the limiting value of the ratio of a circulation line integral surrounding $(x, y)$ to the enclosed area as that area shrinks toward zero:

$$
\mid \text { Curl } F \mid=\lim _{A \rightarrow 0}\left(\frac{1}{A}\right) \oint F \cdot t d s,
$$

where $F$ is the vector function in question (in our case, the thresholded gradient), $t$ is the unit tangential vector, and the circled integration sign indicates integration around a closed path (Schey, 1973).

Not all patterns with subthreshold gradients have inconsistent integrals and curl. In the radial sawtooth of Figure 1, the line integrals are all consistent and the curl is zero at all points. All paths from a point in the inner band to the corresponding point in the outer band must cross the same gradients (Figure 6b).

Patterns with nonzero curl are, however, not rare. The image properties producing curl can be seen more clearly by referring again to Figure 4 . The problem is that

$$
\text { curl } D \neq 0
$$

for some $x$ and $y$, where $D$ is the field of thresholded gradients. Curl $D$ is nonzero where (a)

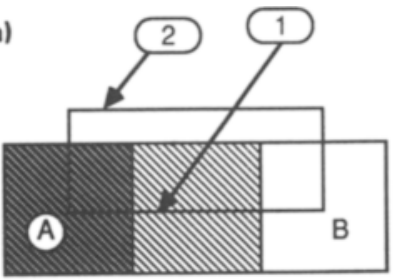

(b)

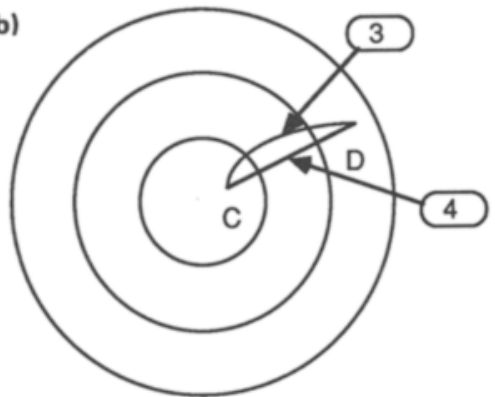

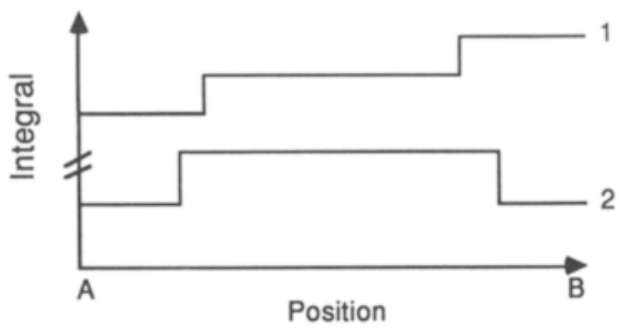

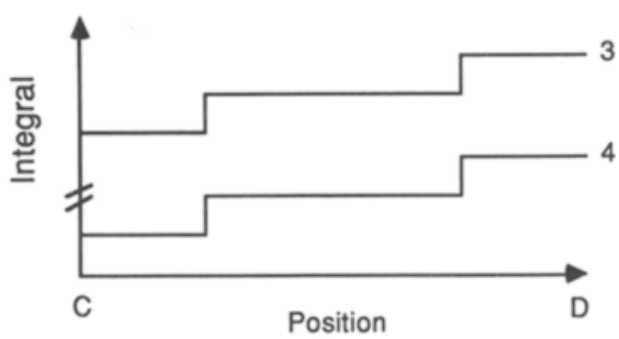

Figure 6. Diagram of patterns with consistent and inconsistent line integrals. (a) Linear sawtooth luminance distribution. Integration along path 1 from point $A$ to point $B$ indicates that $B$ is lighter than $A$. Integration along path 2 says lightnesses at $A$ and $B$ are equal. (b) Radial sawtooth luminance distribution. Integrals along both paths 3 and 4 indicate that $D$ is lighter than $C$. 


$$
E \times \operatorname{grad} \psi \neq 0,
$$

that is, where the gradient of $\log$ retinal illuminance, $E$, and the gradient of the field of thresholding weights, grad $\psi$, are not parallel. This is the case wherever subthreshold or near-threshold illuminance gradients of one orientation are adjacent to steeper illuminance gradients with a different orientation, that is, where there is a twist in the log illuminance surface.

In natural scenes, gradual illumination changes on one spatial dimension are frequently accompanied by steeper changes on the other dimension. For example, such gradients occur when a sidelighted scene is partially occluded by a nearer, differently illuminated surface, and at 3-D intersections of sidelighted and directly illuminated surfaces.

Holding a pattern's contour stationary on the retina prevents encoding of its spatial gradient information. The consequences for spatial integration are similar to those ensuing from thresholding of shallow gradients (Arend, 1973). Curl problems arise in patterns that include both stabilized and unstabilized edges, and in some unstabilized patterns when viewed with natural or simulated scotomas.

Several stabilization experiments are illustrated in Figure 7. An opaque disk is stabilized on the retina so that it occludes a region of an unstabilized pattern. When the subject's fixation is such that eye movements never carry an edge in the unstabilized pattern under the occluder (Figure $7 \mathrm{~b})$, the thresholded gradient distribution $(D$, Figure 4) is perfectly consistent and the integration is

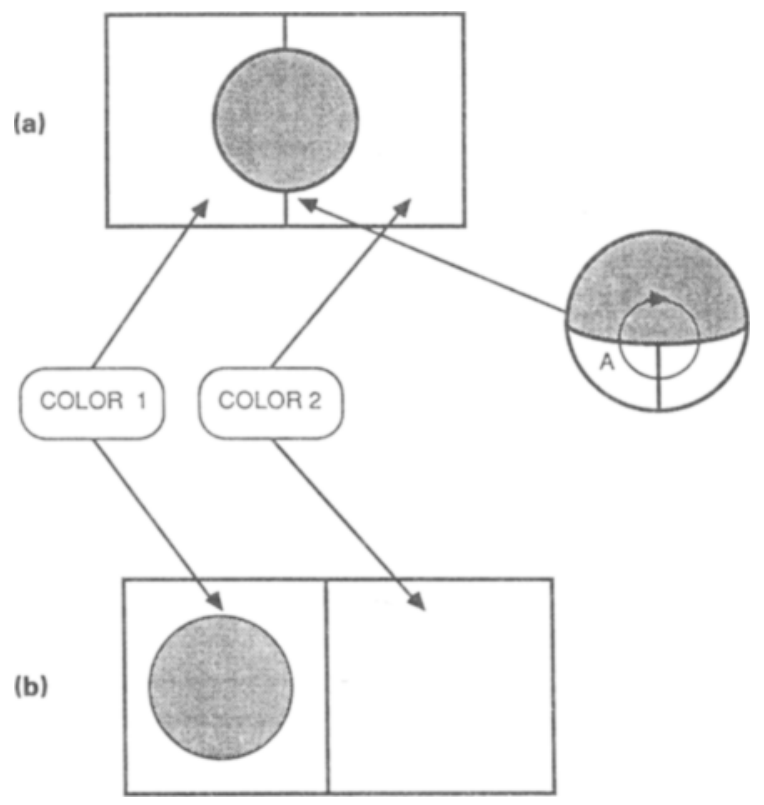

Figure 7. Diagram of experiments with retinally stabilized disk (shaded) occluding part of unstabilized pattern (unshaded). (a) Disk occludes dividing edge of pattern. Inset: Circulation integral at intersection of disk edge and pattern edge. (b) Disk never occludes edges in pattern. straightforward. When the temporal transients from the initial onset of the light have dropped below threshold, the occluded region perceptually fills in according to information from the surrounding unstabilized contour (Gerrits et al., 1966; Krauskopf, 1963; Yarbus, 1967).

When the disk occludes a contour (Figure 7a), the pattern of edge information generated by the subject's eye movements is inconsistent. The gradients in the center of the figure are lost due to the absence of temporal changes in that region as the eye moves. As a consequence, curl $D$ is nonzero at the intersections of the occluder edge and the border (Figure 7a, inset). In this region there are lines of nonzero gradient extending partway across otherwise homogeneous regions of zero gradient. Any circulation integral that encloses an endpoint of one of these lines will be nonzero. In the illustration, the gradient at the point where the path crosses the unstabilized boundary produces an increment in the circulation integral that is not offset by any other change around the path.

There are simple stabilized-image analogues of the radial and linear sawtooth patterns. To our knowledge no one has yet studied such patterns. Figure 8a illustrates the analogue of a two-cycle linear sawtooth luminance distribution and Figure 8b illustrates the analogue of a twocycle radial distribution. The subthreshold shallow luminance gradients within cycles in the sawtooth pattern are here replaced by abrupt (but stabilized) steps. As with the radial sawtooth, the distribution of thresholded gradients in the radial stabilized pattern is perfectly consistent; curl $D$ is everywhere zero despite deletion of the stabilized gradients. The integration should make region $A+B$ uniform and lighter than region $C+D$. In the linear pattern, the gradients along the top and bottom edges are inconsistent with the absence of gradient within each cycle. There is nonzero curl at the ends of the stabilized contours.

\section{Integration Solutions With Curl, Mathematical and Visual}

Prior to Arend's (1985) and Blake's (1985) work, no vision model included explicit analysis of curl or inconsistent integral problems. The human visual system computes a solution in spite of these problems: In most cases patterns with nonzero curl are as stable and determinate in appearance as patterns with no inconsistencies. Very little is currently known about the appearance of patterns that generate curl in the thresholded-gradient field. The remainder of this paper is concerned with what is currently known about those "visual solutions."

\section{Two Visual Solutions}

It is clear from several demonstrations that the visual system's solution involves two very different types of appearance. Linear sawtooth and linear Cornsweet distributions (Figure 6a) have the familiar illusory appearance (invisible luminance gradients within cycles and illusory lightness differences between cycles), but only when the surround luminance differs substantially from the mean 


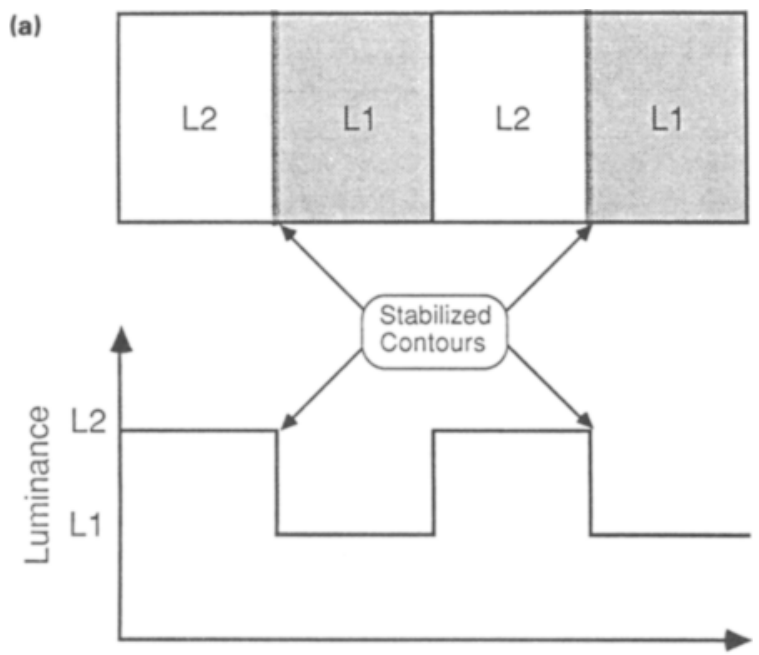

\section{Spatial Position}

(b)

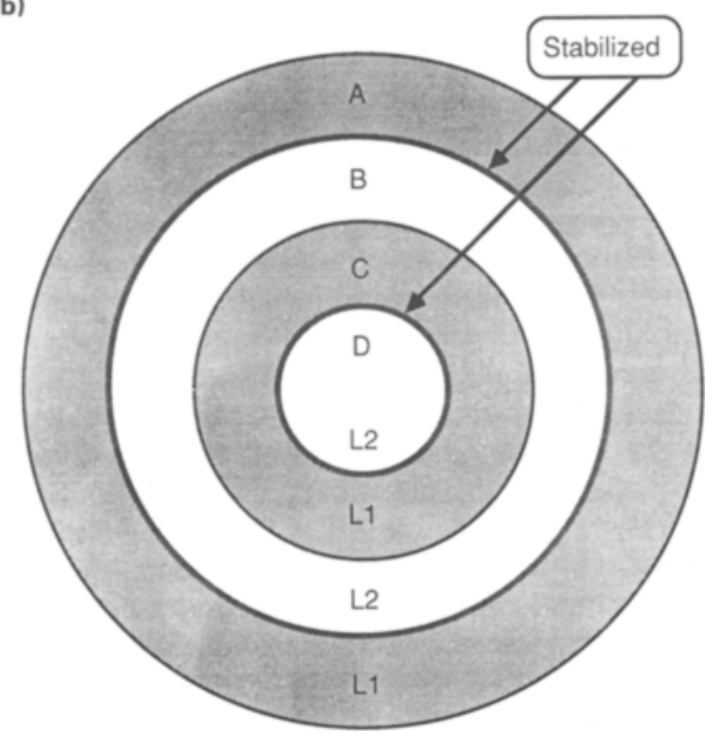

Figure 8. Stabilized retinal image analogues of shallow sawtooth patterns. Stabilization of boundaries between luminances $L 1$ and L2 deletes them from thresholded-gradient field. (a) Linear sawtooth analogue. (b) Radial sawtooth analogue.

luminance. When the surround luminance equals the mean luminance, there is no illusory lightness difference between cycles, and a strong lightness gradient is perceived within each cycle. For any uniform surround, there is curl in the thresholded gradient at the top and bottom edges, and the maximum curl magnitude is independent of surround luminance. The different appearances in these two surround conditions are two distinct outcomes of the visual system's solution to the curl problem. Radial sawtooth and Cornsweet distributions (Figure 6b) (which have zero curl everywhere), on the other hand, produce smooth rings and illusory lightness differences for all surround luminances.

Todorovic (1983) reported a number of interesting gradient illusions. We have studied modified versions of these illusions that give some clues as to how the visual system solves the curl problem. The circumferential sawtooth (Figure 9) is a polar-coordinate version of the sawtooth illusion. The luminance is uniform radially and sawtooth as a function of angle of rotation.

It is immediately obvious that this pattern is problematic for the integration lightness models. A complete clockwise rotation from point $A$ back to point $A$ always crosses the boundaries in the same sense, that is, from dark to light. In the 1-D models, the integral around this path indicates that the lightness at $A$ is not equal to the lightness at $A$ if the shallow gradients are below threshold.

As in the case of the linear sawtooth with mean luminance surround, shallow luminance gradients (smaller than those that appear uniform in the radial sawtooth) do not appear uniform in the circumferential sawtooth. There is no illusory lightness difference between cycles. Unlike the linear sawtooth, however, the circumferential sawtooth has this appearance regardless of the surround luminance.

If one now masks half of the pattern with a sheet of paper, the distribution of curl in the two visible sectors is unchanged. Nevertheless, the observed lightness gradients within the remaining two quadrants are much smaller and the two quadrants have different lightnesses. ${ }^{5}$ The lightness distributions in the top two sectors clearly depend upon the presence of the bottom two sectors.

\section{Field Segmentation}

This two-cycle, half circumferential sawtooth and the linear sawtooth (Figure 6a) on black surround both produce nonzero curi. In these two patterns the sectors differ in lightness and have little lightness gradient within sectors. Their appearance is an example of the visual solution we call field segmentation, for which we know of no existing computational algorithm.

The lightnesses perceived within the sawtooth are consistent with the pattern of thresholded gradients between (but not including) the inner and outer borders of the annulus. They are not consistent with the thresholded gradients at the annulus borders, which indicate different lightnesses at the peak and trough of the sawtooth. The visual system apparently resolves the contradiction by ignoring the variation in the thresholded gradient around those borders.

Curl is often confined to a few locations within the field. For example, Figure 10 shows calculated curl distributions (algorithm in Appendix) in the thresholded gradient distributions of the circumferential sawtooth patterns at two surround luminances. Curl is restricted to the inner and outer borders of the annulus.

The appearance of these patterns suggests that the visual system adopts the following integration algorithm: Parti- 


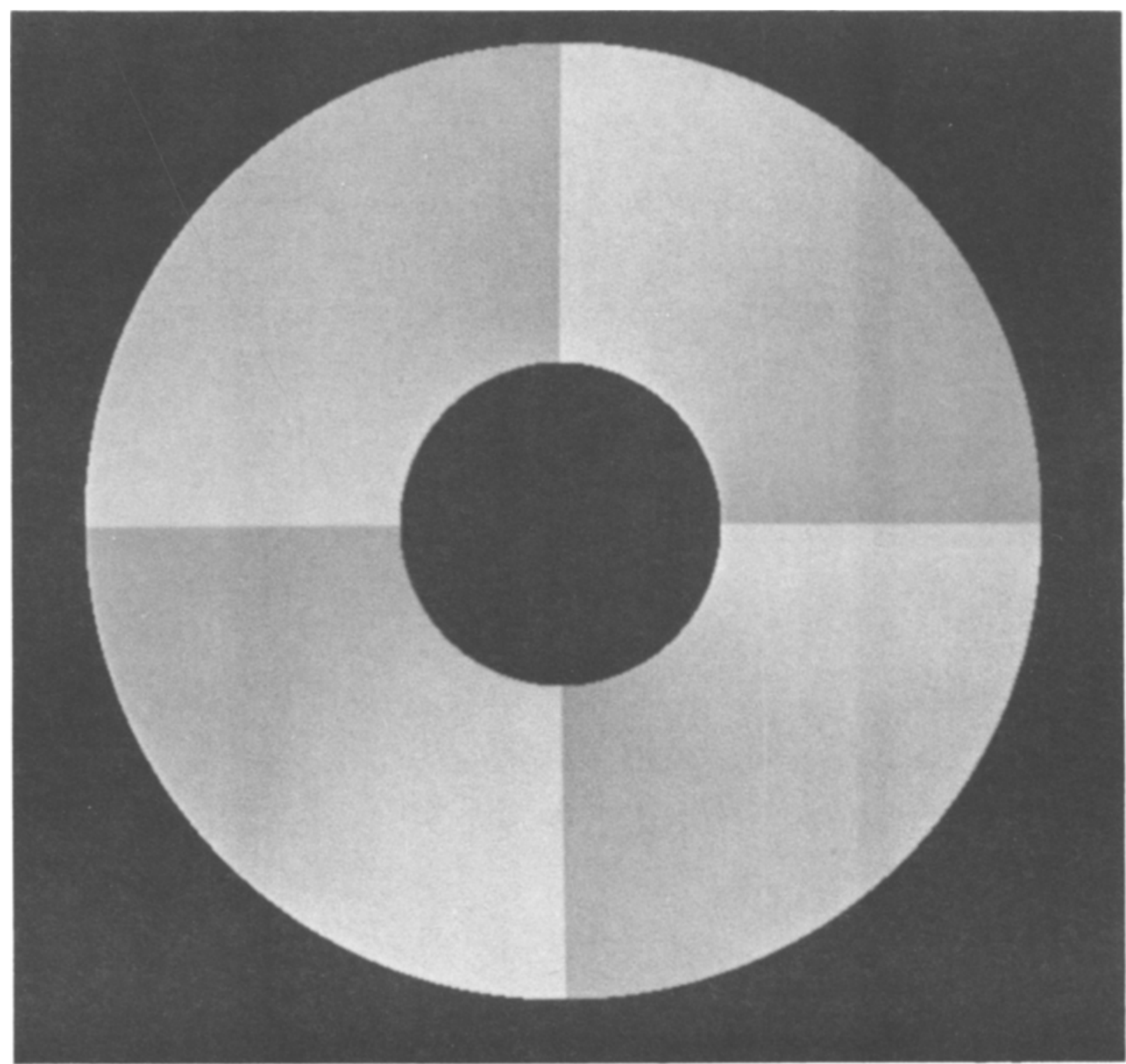

Figure 9. Circumferential sawtooth pattern. The luminance distribution is four identical cycles of sawtooth around the circumference of the annulus.

tion the visual field into domains in such a way that curl points are on the edges of domains (and excluded). Then integrate separately within the resulting curl-free domains. Stokes's theorem (Schey, 1973) states that closed paths that enclose no points with nonzero curl have circulation integrals equaling zero. Within a single-bounded, curlfree domain there are no inconsistent line integral problems, and the thresholded-gradient distribution is itself the gradient of a pattern.

This segmentation problem should not be confused with the more familiar image-segmentation problem of trying to identify boundaries of objects and other real-world entities (see Rosenfeld \& Kak, 1982, chap. 10, for an over- view). The useful features of global integration are lost if the integration domains are not defined as large as the curl problem permits. We have not yet attempted to formulate a computational scheme segmenting on the basis of curl. Segmentation algorithms developed for other purposes will have to be modified for this purpose if they are to preserve the desirable global features of the integration lightness models. Once curl-free domains are identified, a region-growing version of the algorithm in the Appendix can be applied within domains.

In the two-quadrant circumferential sawtooth pattern, the "bent rectangle" defined by the border of the half annulus contains no curl. When the sawtooth's shallow gra- 

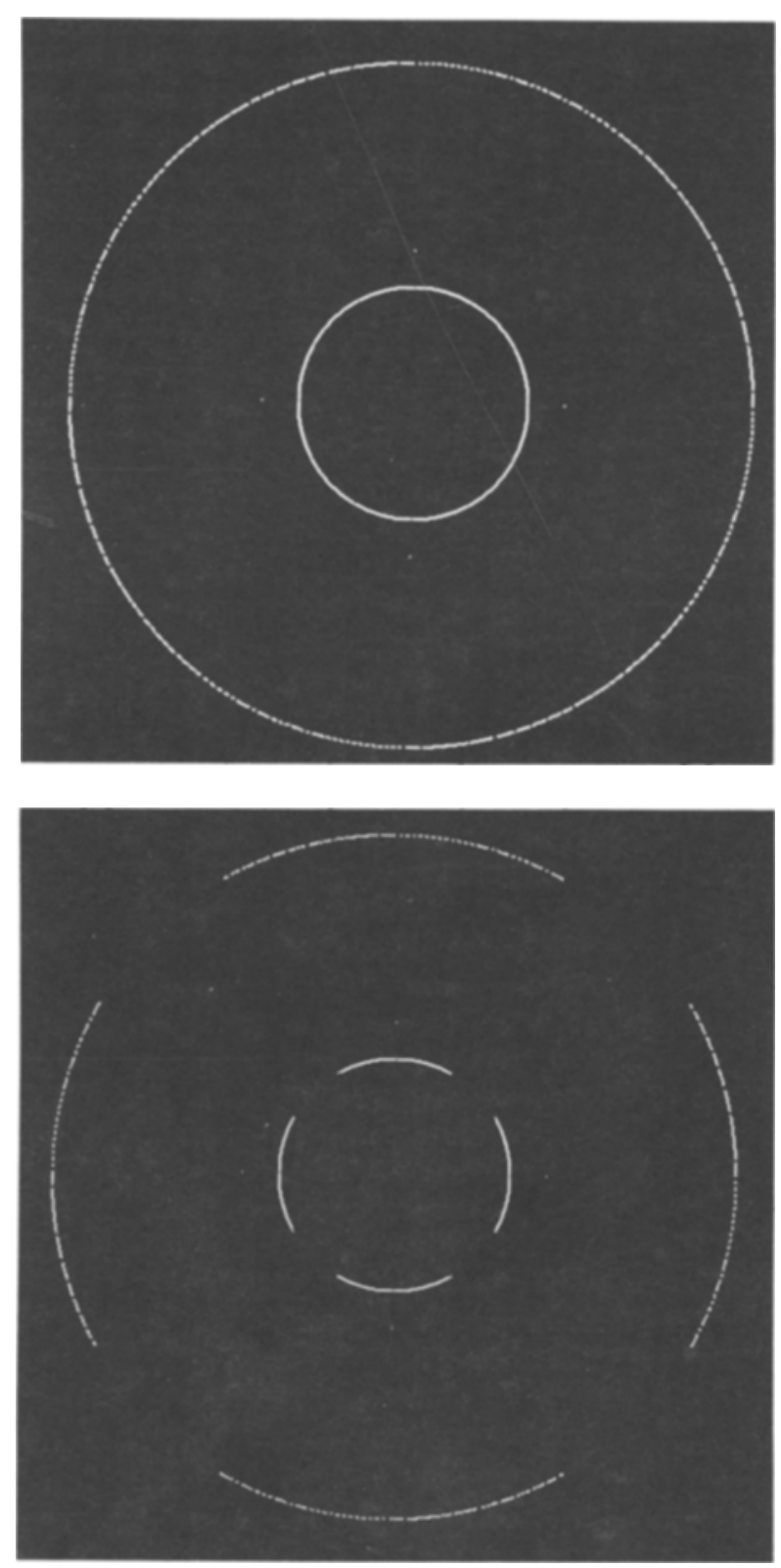

Figure 10. Calculated curl field for circumferential sawtooth pattern on dark surround and on mean-luminance surround. Increasing curl magnitude is indicated in figure by increasing lightness. Curl is confined to inner and outer borders of sawtooth annulus.

dient is subthreshold, the thresholded gradient is identical to that for a step distribution, and a clear illusory lightness step occurs.

In the four-quadrant circumferential sawtooth pattern, there are no illusory lightness differences, and strong lightness gradients are perceived within sectors. The visual system apparently cannot segment the field into singlebounded, curl-free areas. The annular sawtooth region is curl-free, but its outer boundary encloses the curl at the inner boundary. One might try to solve this problem by arbitrarily designating the boundary between any two of the sectors as an end boundary, ignoring the information at that boundary (which contradicts the lightness relation indicated by the integral over the enclosed space) (Figure 11). The integration would then be similar to that for the half distribution. A four-step lightness illusion would occur with the selected boundary flanked by the lightest and darkest regions. Since this illusion does not occur, we conclude that the visual system cannot arbitrarily designate such a contour as the boundary of an integration domain.

The visual system's rules for establishment of a domain boundary have not yet been empirically studied. In our
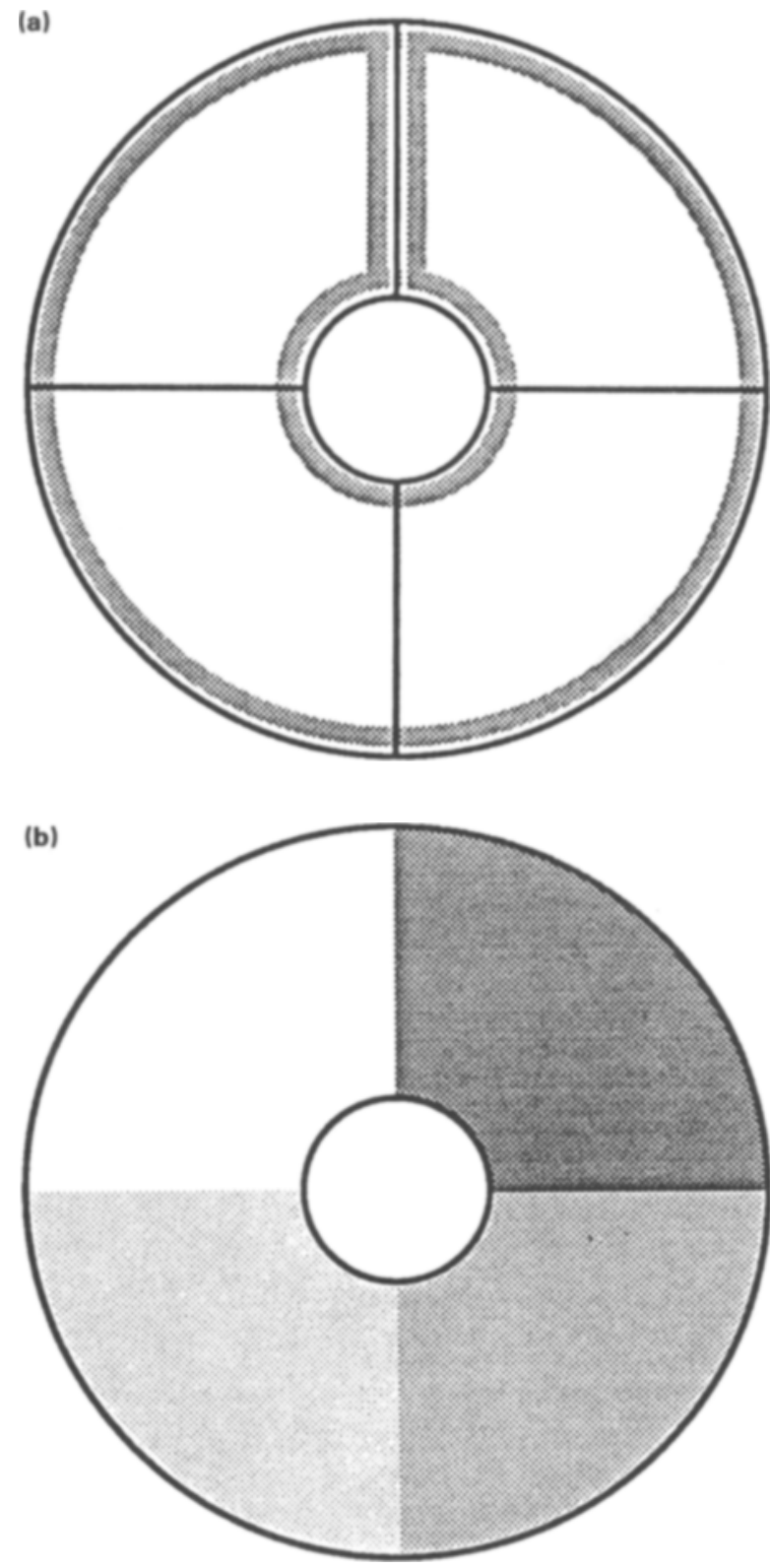

Figure 11. (a) Boundary (shaded) on circumferential sowtooth enclosing zero-curl area. The pattern of thresholded gradient within the shaded border is identical to that from (b) a four-step hminance distribution. 
informal explorations of the four-cycle case, we have found that simply drawing a thin black line along one of the contours between quadrants does not establish a visual domain boundary. So far the only manipulation we have found sufficient is separation of the sectors by a substantial area of contrasting background.

\section{Lightness Gradient Manipulation}

When the surround luminance equals the mean luminance of the linear sawtooth or the circumferential sawtooth, the pattern passes through the surround luminance in the middle of each cycle. The inner and outer contours of the ring vanish in those regions. Strong lightness gradients occur within cycles and there is no illusory lightness difference between cycles. This appearance indicates that the visual system cannot segment the field under these conditions, perhaps due to the absence of a closed physical contour along the lines where curl is nonzero. In these cases a second kind of curl solution is applied.

The solution appears to be of a type we will call gradient manipulation. In these algorithms the problematic thresholded-gradient field is replaced by a field that has zero curl everywhere. Blake (1985) presented a gradientmanipulation algorithm that is a refinement of Horn's (1974) approach. In Blake's algorithm the thresholded gradient is differentiated again to provide a second-derivative field similar to Horn's thresholded Laplacian field, that is, $\operatorname{div} D$.

Blake then applied an improved double-integration algorithm, based on Gauss-Seidel relaxation, to give the predicted lightness field. The algorithm is based on a continuous solution that evenly distributes over the predicted lightness field the perturbations required to eliminate the curl. When there is curl in the thresholded gradients, the result is that the lightness gradients in the output have no simple relationship to luminance gradients at the corresponding positions in the input image, but they usually will not deviate far from what the local luminance gradients indicate.

A second, relatively simple, gradient-manipulation solution to the curl problem is based on the fact that the curl is a consequence of loss of gradients in the threshold process. One could compute curl in the thresholded gradient and feed the results back on to the threshold operator, manipulating the threshold function to eliminate curl. This algorithm would make the model's threshold for local gradients of $\log$ illuminance a function of global characteristics of the input pattern. Development of this algorithm is at an early stage.

It is useful in analyzing the visual properties of these solutions to consider a model's computed output, the lightness field, as having two kinds of lightness gradients. Define vector fields $F(x, y)$ and $G(x, y)$ such that

$$
\boldsymbol{G}=\boldsymbol{F}-\boldsymbol{D}
$$

where $F$ is the gradient of the computed lightness field and $D$ is again the thresholded gradient of log retinal illuminance. $G$ is thus a field of computational illusory gra- dients, the spatial lightness changes the integration algorithm must add to the stimulus information in order to eliminate the curl in $\boldsymbol{D}$. It is always possible to find such a $G(x, y)$. One type of solution, then, to the problem that $D$ is not a gradient field is to add to $D$ a vector field that produces a gradient field and then integrate.

If the $\boldsymbol{G}$ found by the algorithm is a monotonic increasing function of the component of grad $\log H$ that was removed by the threshold operator, the resulting percept is veridical in the sense that lightness gradients are perceived only where there are corresponding gradients of retinal illuminance. For any other $\boldsymbol{G}$ the result of the integration is illusory; some of the lightness gradients in the output bear no simple relationship to $\log H$ gradients at the corresponding location in the input pattern. An example of such an illusory situation is shown in Figure 12. A central uniform rectangle is surrounded by a rectangular annulus containing a shallow luminance gradient. When the gradient is subthreshold there is curl along the top and bottom edges of the central rectangle. The visual system's solution is a lightness pattern with a uniform annulus. The central rectangle contains shallow lightness gradients that are illusory in the above sense.

The visual system also solves integration problems for some stabilized patterns by gradient manipulation. For the pattern illustrated in Figure $7 \mathrm{~b}$, the subject sees an illusory gradient of lightness and color over the occluded region, smoothly joining the centers of the two squares (Gerrits et al., 1966; Yarbus, 1967). The gap in the contour apparently prevents any kind of field-segmentation solution.

The experimentally observed threshold for perceiving a lightness gradient (whether veridical or illusory) clearly depends upon whether the field-segmentation appearance occurs. For example, covering one quadrant of Figure 9 produces an open three-cycle pattern with the appearance we have labeled field segmentation. Very little lightness gradient is perceived within sectors. When three cycles of the same physical contrast extend around a full circle, the gradient-manipulation appearance occurs. Large lightness gradients are perceived within the cycles, even though the luminance gradient is $25 \%$ smaller than the subthreshold gradient in the open three-cycle pattern.

\section{Conditions for Field Segmentation}

Of the two types of solutions to curl problems, field segmentation is apparently the higher priority solution. The gradient-manipulation solution is always available to the visual system. For example, the top half of the circular sawtooth need not change appearance when the bottom half is masked. The drastic change of appearance when field segmentation is possible contradicts the predictions of models (e.g., Horn's [1974] and Blake's [1985]) that always apply the gradient-manipulation solution. They therefore require modification if they are to serve as models of human lightness perception.

At least two types of problems can prevent field segmentation. When the circumferential sawtooth is sur- 

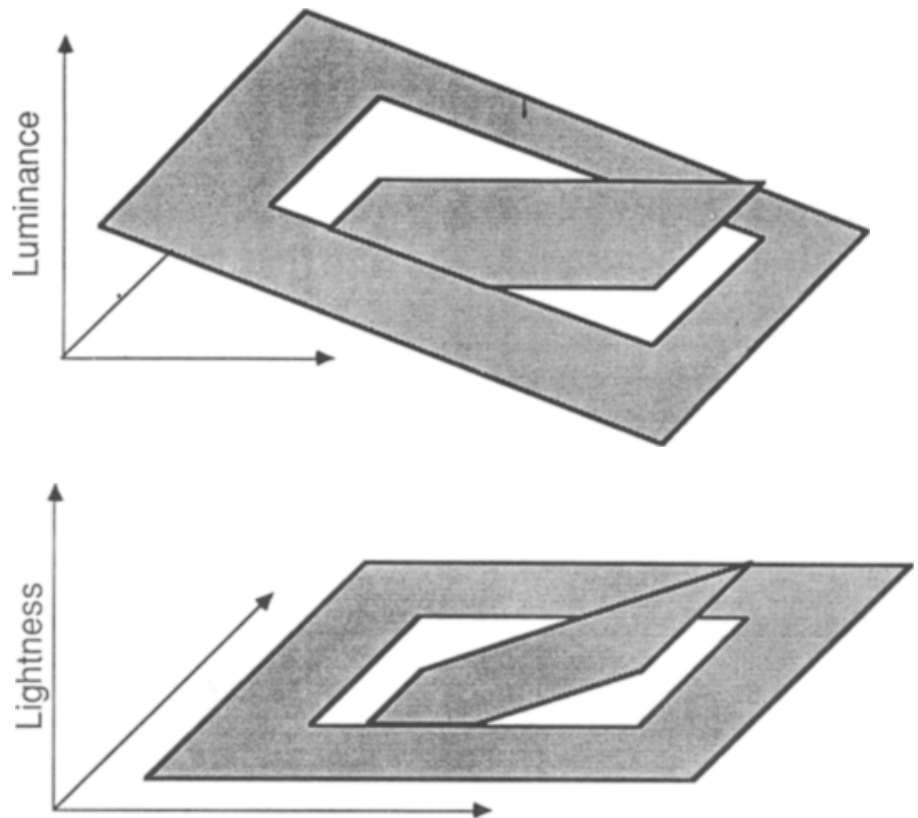

Figure 12. Diagram of shallow gradient luminance pattern and its appearance, an illusory lightness pattern. The uniform-luminance central rectangle has a clear lightness gradient, whereas the luminance gradient in the surrounding rectangle is not perceived. This is an example of a gradient-manipulation solution with illusory placement of lightmess gradients.

rounded by the mean luminance, there are gaps in the inner and outer contours of the annulus and corresponding gaps in the curl distribution. It is intriguing that one does not obtain the kind of contour completion phenomena observed with other types of incomplete figures (e.g., the Kanizsa triangle), especially considering that the gaps prevent the preferred segmentation solution. It is readily demonstrated that physically completing the contour produces the segmentation solution. For example, a narrow black line drawn around the edge of the two-sector pattern restores the illusion.

In the four-segment sawtooth, the segmentation solution is prevented by the topology of the curl sites rather than inadequate local boundary contrast; the inner border is completely enclosed within the outer border. In this pattern, no nonarbitrary segment boundary can be formed to link the inner and outer borders, preventing partition of the field into curl-free domains.

\section{EXPERIMENT}

As a first effort in the quantitative study of curl solutions, we measured lightness differences in circumferential sawtooth patterns. We asked our subjects to adjust the luminance of a small square in the comer of our display to match the lightness at the center of each of the sawtooth quadrants. We used the full and half patterns on several background luminances. The data confirm the qualitative appearance of Figure 9 discussed above, reflecting field segmentation when possible and lightness gradient manipulation otherwise.
Subjects

Two subjects were used: one of the authors (L.A.) and a paid volunteer (A.R.), who was unaware of the purpose of the experiment.

\section{Display}

The patterns used are illustrated in Figure 9. The circumferential sawtooth consisted of four cycles of a polar coordinate sawtooth luminance distribution with angular frequency $4 \mathrm{c} / 2 \pi, 0.20$ contrast, and mean luminance $38 \mathrm{~cd} / \mathrm{m}^{2}$. Inner and outer diameters of the circumferential sawtooth were $5.0^{\circ}$ and $15.0^{\circ}$ of visual angle, respectively. The matching stimulus was a $2.0^{\circ}$ square $18.4^{\circ}$ from the display center and separated from the background by a $1.0^{\circ}$-wide zero-luminance border.

Patterns were displayed on a Tektronix 690 RGB monitor. Patterns were calculated on a VAX 11/750 computer and stored in an Adage 3000 image processor. The image processor generated a $512 \times 512$ pixel, $60-\mathrm{Hz}$ noninterlaced image with 10 -bit resolution on each color gun. The display system was fully calibrated for luminance and chromaticity, correcting for monitor nonlinearity with lookup tables. Details of the display system and calibrations are presented elsewhere (Arend \& Reeves, 1986).

\section{Procedure}

Each subject viewed the patterns binocularly from an ophthalmic chin- and headrest $51 \mathrm{~cm}$ from the screen. Because the patterns were large, the subject was allowed to look back and forth freely from the sawtooth to the matching rectangle.

On each trial the subject adjusted the luminance of the rectangle to match the lightness at the center of one of the quadrants. The surround luminance and the quadrant to be matched were pseudorandomly varied over trials. The matching luminance was controlled by the horizontal position of a bitpad cursor. Endpoints of the adjustment were randomly offset between trials to avoid position cues. 


\section{Results}

The left panels in Figures 13 and 14 show the matching data for the two-quadrant condition, and the right panels show the four-quadrant data. Matches to the upper two quadrants are in the top panels, and the lower panels show matches to the lower two quadrants.

For the first subject (L.A.; Figure 13), substantial illusory lightness differences occurred between the centers of the right and left quadrants when the surround luminance was greater than the maximum luminance of the sawtooth or less than the minimum luminance. When the surround luminance equaled the mean luminance of the
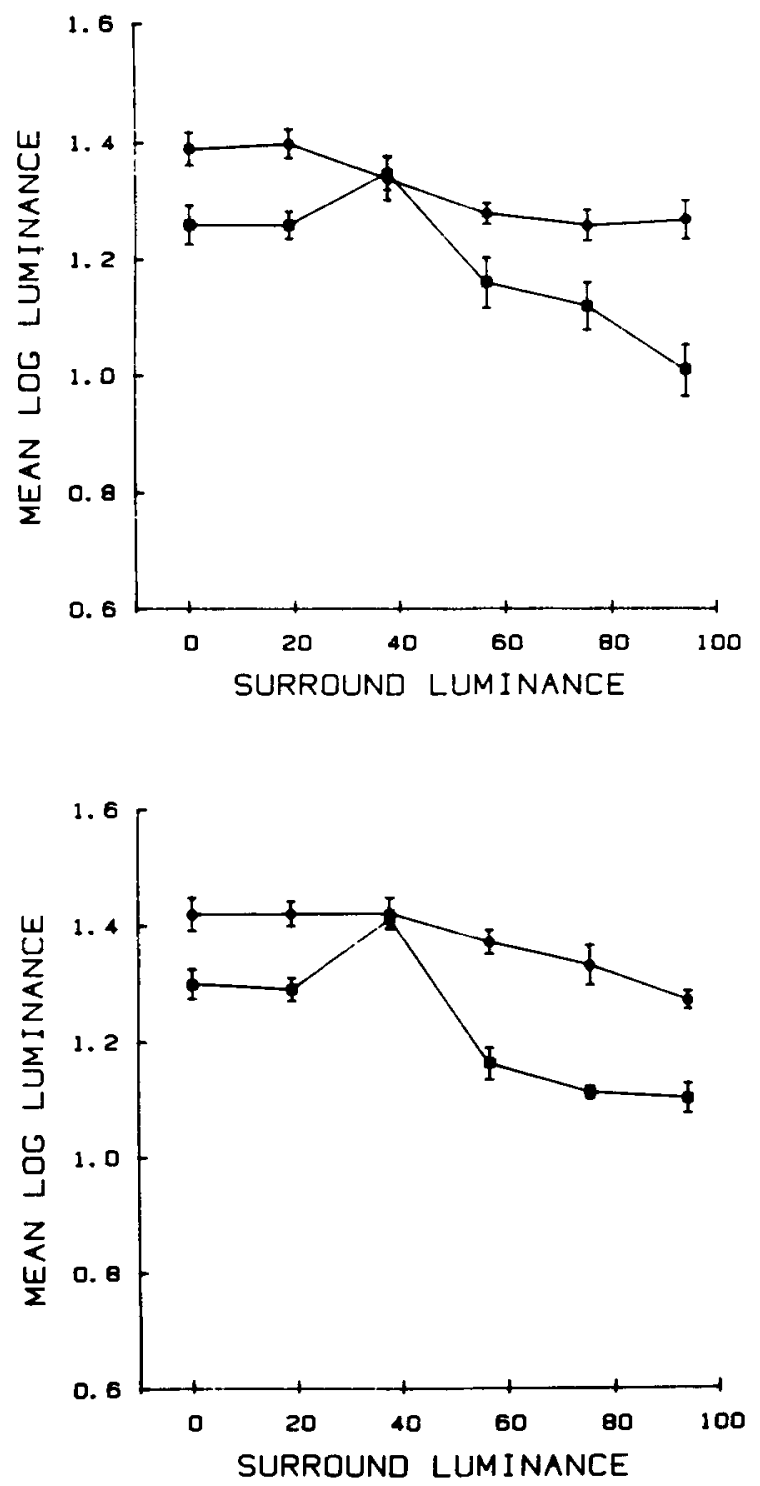

sawtooth $\left(38 \mathrm{~cd} / \mathrm{m}^{2}\right.$, third point from left), there was no illusory lightness difference between quadrants. When all four quadrants of sawtooth were present, the lightness differences were much smaller. (Several of the differences are reversals, in the direction opposite the expected illusion.) The data therefore agree with the subjective appearance of the patterns in Figure 9.

Results for the second subject (A.R.; Figure 14) were the same for the bottom quadrants, but for the top quadrants he matched the left quadrant lighter than the right throughout. The difference was least with the meanluminance surround. The difference was greater for the
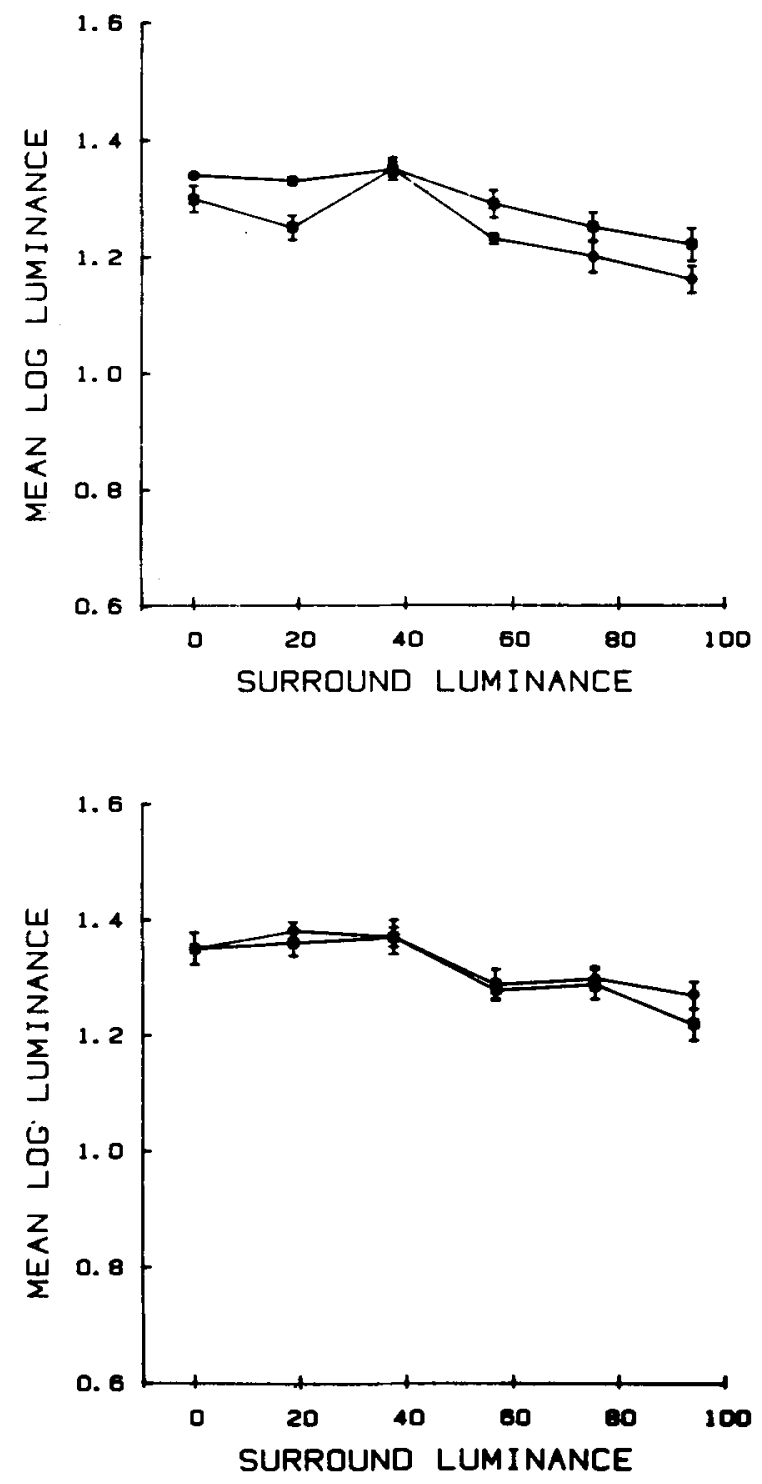

Figure 13. Lightness matching data for circumferential sawtooth patterns (Subject L.A.). Mean log match luminance (cd/m²) as a function of surround luminance $\left(\mathrm{cd}^{\mathrm{m}} \mathrm{m}^{2}\right)$. Peak, mean, and trough luminances of sawtooth are 30.4, 38.0, and $45.6 \mathrm{~cd} / \mathrm{m}^{2}$, respectively. Error bars are \pm one standard error of the mean. Left: Half annulus (two cycles). Right: Full annulus (four cycles). Top: Upper two quadrants. Bottom: Lower two quadrants. Circles in each panel are for quadrant predicted to be lighter. 

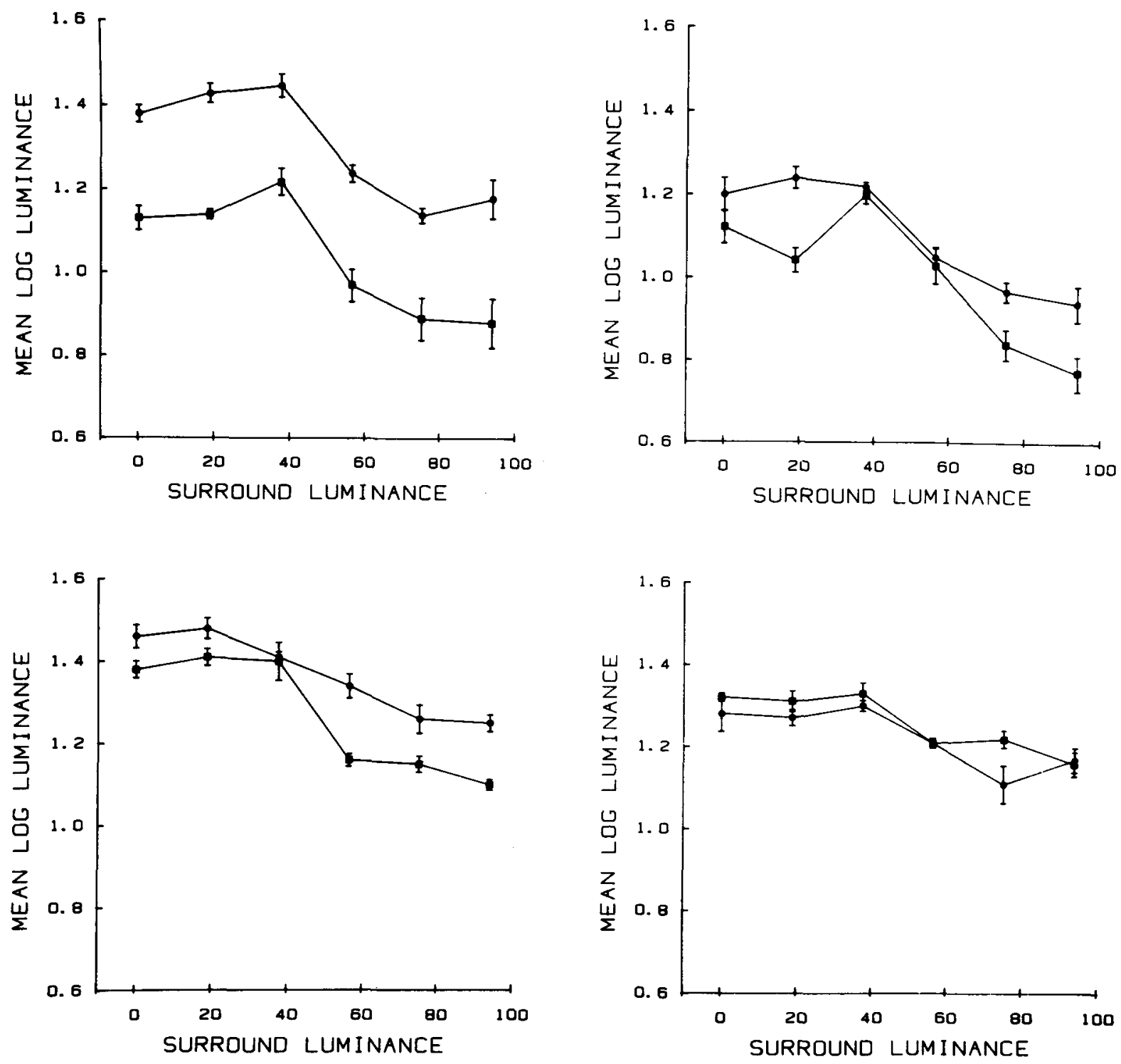

Figure 14. Lightness matching data for circumferential sawtooth patterns (Subject A.R.).

half ring than for the full ring. The absence of differences for the bottom quadrant suggests that A.R.'s top matches differ from those of L.A. due to a response bias.

\section{DISCUSSION}

Our model is currently incomplete. The demonstrations and matching data show that the visual system prefers the field-segmentation solution when both field segmentation and lightness gradient manipulation are possible. They also show that there are at least two factors that can prevent field segmentation: failure to form a segmenting contour and topological problems in potential segmenting contours. We have only qualitatively described the conditions necessary for the field-segmentation percept to occur, and there are probably others to be discovered. A quantitative algorithm for the gradient-manipulation solution must also be developed and empirically tested. Experimental progress on both issues is likely to be severely hampered by the lack of widely tested and accepted methods for psychophysically describing gradual spatial changes of lightness.

\section{REFERENCES}

AREND, L. E. (1973). Spatial differential and integral operations in human vision: Implications of stabilized retinal image fading. Psychological Review, 80, 374-395.

AREND, L. E. (1976a). Response of the human eye to spatially sinusoidal gratings at various exposure durations. Vision Research, 16, 1311-1315. 
AREND, L. E. (1976b). Temporal determinants of the form of the spatial contrast threshold MTF. Vision Research, 16, 1035-1042.

AREND, L. E. (1985). Spatial gradient illusions and inconsistent integrals. Investigative Ophthalmology \& Visual Science, 26, 280.

AREND, L. E. (1987). Cornsweet illusion without subthreshold luminance gradients. Manuscript in preparation.

Arend, L. E., Buehler, J. N., \& Lockhead, G. R. (1971). Difference information in brightness perception. Perception \& Psychophysics, 9, 367-370.

AREND, L. E., \& ReEVES, A. (1986). Simultaneous color constancy. Journal of the Optical Society of America A, 3, 1743-1751.

BLAKE, A. (1985). Boundary conditions for lightness computation in Mondrian world. Computer Vision, Graphics \& Image Processing, 32, 314-327.

Campbell, F. W., Howell, E. R., \& Robson, J. G. (1971). The appearance of gratings with and without the fundamental Fourier component. Journal of Physiology, 217, 17P-19P.

Cohen, M. A., \& GrossberG, S. (1984). Neural dynamics of brightness perception: Features, boundaries, diffusion, and resonance. $P$ erception \& Psychophysics, 36, 428-456.

CORNSWEet, T. N. (1970). Visual perception. New York: Academic Press.

CRAIK, K. J. W. (1966). The nature of psychology: A selection of papers, essays, and writings. Cambridge, MA: University Press.

Davidson, M., WHITEsIDE, J. A. (1971). Human brightness perception near sharp contours. Journal of the Optical Society of America $A, 61,530-536$.

DoOLeY, R. P., \& GRENFIELD, M. I. (1977). Measurements of edgeinduced visual contrast and spatial-frequency interaction of the Cornsweet illusion. Joumal of the Optical Society of America, 67, 761-765.

Evans, R. M. (1974). The perception of color. New York: Wiley.

FrY, G. A. (1948). Mechanisms subserving simultaneous brightness contrast. American Journal of Optometry, 25, 162-178.

Gerrits, H. J. M., DE HAAN, B., \& VendrIK, A. J. H. (1966). Experiments with retinal stabilized images: Relations between the observations and neural data. Vision Research, 6, 427-440.

Gilchrist, A., Delman, S., \& Jacobsen, A. (1983). The classification and integration of edges as critical to the perception of reflectance and illumination. Perception \& Psychophysics, 33, 425-436.

HoRn, B. K. P. (1974). Determining lightness from an image. Computer Graphics \& Image Processing, 3, 277-299.

KRAUSKOPF, J. (1963). Effect of retinal image stabilization on the appearance of heterochromatic targets. Joumal of the Optical Society of America $A, 53,741-744$.

LAND, E. H. (1986). Recent advances in retinex theory. Vision Research, 26, 7-22.

LAND, E. H., \& MCCANN, J. J. (1971). Lightness and retinex theory. Journal of the Optical Society of America, 61, 1-11.

O'BRIEN, V. (1958). Contour perception, illusion, and reality. Journal of the Optical Society of America, 48, 112-119.

RatufF, F., \& SiRovich, L. (1978). Equivalence classes of visual stimuli. Vision Research, 18, 845-853.

RosenFeLD, A., \& KAK, A. C. (1982). Digital picture processing (2nd ed.). New York: Academic Press.

SCHEY, H. M. (1973). Div, grad, curl, and all that. New York: Norton.

ToDorovic, D. (1983). Brightness perception and the Craik-O'BrienCornsweet effect. Unpublished master's thesis, University of Connecticut, Storrs.

WaLLS, G. L. (1954). The filling-in process. American Journal of Optometry, 31, 329-341.

Yarbus, A. L. (1967). Eye movements and vision. New York: Plenum Press.

\section{NOTES}

1. We will not be directly concerned in this paper with the distinction between lightness and brightness (see Evans, 1974, for definitions); for convenience we will use the term lightness to refer to both. Although it is the stated goal of most of the models discussed in this paper to predict lightness, a perception of surface reflectance, none of the models considered herein can distinguish between a sharp reflectance edge and a sharp illumination edge. They are not, therefore, in themselves sufficient models of lightness constancy.

2. For our present concerns it is irrelevant whether the sensitivity profile of the visual filter is due to the response of a single broad bandpass filter or is the envelope of sensitivities of multiple medium-bandwidth filters. At least for purposes of conscious representation of spatial patterns, the visual system combines the outputs of any frequency-selective analyzers. There is no parallel conscious representation of activity in narrow-frequency bands like that in the auditory system (Ohm's acoustic law).

3. Gilchrist, Delman, and Jacobsen (1983) argued from lightness constancy data that integration of edge information must occur simultaneously, in parallel, in lightness and perceived-illumination distributions. We will not address here the constancy issue of how the visual system manages to separate spatial retinal illumination changes into reflectance changes and illumination changes. For the sake of simplicity we will confine our discussion to uniformly illuminated flat matte surfaces and, therefore, to spatial changes of reflectance. Most of the discussions are applicable to spatial illumination changes, once the constancy problem is solved. The algorithms discussed here may contribute to constancy solutions, but they do not, in themselves, provide lightness or color constancy.

4. One possible exception to this is Cohen and Grossberg's (1984) extension of Grossberg's neural network model to gradient illusions. Their basic filling-in mechanism is local; that is, contour signals diffuse locally, within contours. Their simulations qualitatively match serial illusory lightness distributions, including a version of the Cornsweet illusion (their Figure 7). In this simulation, three regions of the same luminance, separated by two Cornsweet edges, correspond to three differnt lightnesses. This result is quite sensitive, however, to the contrast at the outer edges of the pattern, where the rightmost and leftmost of the three regions meet the surround. Our experience with three-cycle gradient illusions (e.g., Figure 1), on the other hand, is that the illusory appearance of the patterns does not strongly depend on the surround conditions. The three rings of Figure 1 have approximately the same appearance either on the black surround shown or on a white surround. (The skeptical reader may confirm this by covering the black surround with a piece of heavy white paper with a hole cut in it.) Nevertheless, unlike earlier local filling-in models, this model is sufficiently complex that it may be able to account for a wide variety of gradient illusions, with suitable adjustments.

5 . By carefully fixating from a short viewing distance one of the contours of the unmasked Figure 6, it is sometimes possible to see an illusory lightness difference between the adjacent regions. A large eye movement will usually abolish the lightness difference and produce lightness gradients within the sectors. This effect is probably attributable to placement of the conflicting information in the periphery, simulating the effects of masking off two of the quadrants (Yarbus, 1967, p. 79).

\section{APPENDIX \\ Details of Model}

\section{Gradient}

For the gradient calculation, we use as discrete approximations of the partial derivatives in the $x$ and $y$ directions

$$
d_{x}(i, j)=L(i, j+1)-L(i, j)
$$

and

$$
d_{y}(i, j)=L(i+1, j)-L(i, j)
$$

where $L(i, j)$ is the data value of the pixel in row $i$ and column $j$, that is, the logarithm of retinal illuminance at position $i, j$. 
Our discrete approximation of the magnitude of the gradient is then given by

$$
G(i, j)=\left[d_{x}(i, j)^{2}+d_{y}(i, j)^{2}\right]^{1 / 2}
$$

and the direction by

$$
T(i, j)=\tan ^{-1}\left(\frac{d_{y}(i, j)}{d_{x}(i, j)}\right) .
$$

\section{Threshold}

The gradient field is thresholded by multiplying the $G(i, j)$ at each point $i, j$ by a scalar weight, $W(i, j) . W(i, j)$ is a tabled function of $G(i, j)^{2}$, the squared magnitude of the gradient; $W$ increases in a sigmoid form from zero to one.

The magnitude of the thresholded gradient is thus

$$
\underline{G}(i, j)=W(i, j) G(i, j)
$$

where the underscore indicates the thresholded quantity. The direction of the thresholded gradient is the same as that of the unthresholded gradient. The $x$ and $y$ components of the thresholded gradient are

$$
\underline{d}_{x}(i, j)=W(i, j) d_{x}(i, j)
$$

and

$$
\underline{d}_{y}(i, j)=W(i, j) d_{y}(i, j)
$$

For the calculation of Figure 5, we used a step distribution for $W(i, j)$; that is, the weight was zero below the threshold magnitude and one above. We have not yet attempted to empirically obtain a $W$ function closely fitting psychophysical data.

\section{Integration}

To integrate over the entire image we integrate along columns of thresholded $y$ partial derivatives, obtaining the relative column values from a row of thresholded $\boldsymbol{x}$ partial derivatives:

$$
R(i, j)=R_{0}+\sum_{n=1}^{j-1} \underline{d}_{x}(1, n)+\sum_{m=1}^{i-1} \underline{d}_{(}(m, j)
$$

where $R(i, j)$ is the predicted lightness at position $i, j$ and $R_{0}$ is the lightness of pixel $(1,1)$, arbitrarily chosen at present. When there is no curl, this algorithm is sufficient to produce predicted lightnesses that approximate the observed appearance of the pattern. When curl is present, the global inconsistencies in the thresholded derivative information produce errors that propagate down columns, producing streaks that are not visible in the perceived pattern.

Curl

Our estimate of the magnitude of the curl of the thresholded gradient field is a discrete approximation derived from the circulation definition of curl magnitude. The continuous expression is

$$
|\operatorname{Curl} F|=\lim _{A \rightarrow 0}\left(\frac{1}{A}\right) \phi F \cdot t d s,
$$

where $F$ is the thresholded-gradient vector at a point on a closed path of integration enclosing point $i, j ; t$ is the unit tangent vector to the path at the same point; $A$ is the area enclosed by the path; and $s$ is distance along the path.

We approximate this quantity by summing thresholded gradient components around a small, closed ring of pixels enclosing point $i, j$. To avoid orientation artifacts due to the finite size of our enclosed area, we need to define our ring of pixels as nearly circular as is practical. To accomplish this we found it necessary to do the curl calculation on a hexagonal pixel grid, rather than the rectangular grid of the rest of the model. The first pixel in every second row of pixels was sampled one-half pixel to the left in the picture, giving each pixel six roughly equidistant neighbors, two each in the rows above and below, and one each on the right and left in the same row.

The magnitude of the curl at pixel $i, j$ is approximately the sum of the threshold differences between data levels around the ring of $i, j$ 's six neighbors:

$$
C=\sum_{n=2}^{6} W_{n}\left(L_{n}-L_{n-1}\right)+W_{1}\left(L_{1}-L_{6}\right),
$$

where $L_{1}$ through $L_{\delta}$ are the pixel data at the six neighbors and $W_{n}$ is the tabled threshold-weighting-function value for $\left(L_{n}-L_{n-1}\right)^{2}$.

(Manuscript received August 8, 1986; revision accepted for publication February 9, 1987.) 